Pacific Journal of Mathematics

ON THE ESSENTIAL SPECTRUM OF THE HYDROGEN 


\title{
ON THE ESSENTIAL SPECTRUM OF THE HYDROGEN ENERGY AND RELATED OPERATORS
}

\author{
P. A. Rejto
}

Titchmarsh determined the spectrum of the Schrödinger energy operator associated with the hydrogen atom, i.e. the operator $-\Delta-1 / r$. He showed, in particular, that its essential spectrum consists of the positive real axis. On the other hand, Agudo-Wolf and Birman formulated overlapping criteria for a potential, which ensured that addition of such a potential does not change the essential spectrum of $-\Delta$.

These criteria do not admit the potential $1 / r$ and a criterion admitting it is formulated in the forthcoming work of Balslev where he also considers operators in $L_{p}$ spaces. In this paper we slightly extend this Balslev criterion, in case the operator is a Schrödinger operator. Our proofs are different, inasmuch as we capitalize on the representation of the kernel of the unperturbed resolvent. Then we make essential use of a result of Friedrichs which gives a bound for the norm of an integral operator.

A criterion which ensures that the essential spectra of two self adjoint operators are equal is the following: for an appropriate complex number $\zeta$ the difference of their resolvents is compact. We shall refer to this property by saying that these operators are resolvent congruent. This property requires less and ensures less, then the one introduced independently by de Branges [18] and Birman-Krein [11] in connection with the perturbation of the continuous spectrum. It is implied by one which we called relative compactness by Gokhberg-Krein [24] and for convenience this is shown in $\S 1$. For semi-bounded operators still another property was introduced by Birman [9] who showed that his property also implies this one.

In $\S 1$ we recall the classical notion of the essential spectrum, and formulate some general operator theoretic facts, which are used in the subsequent sections. Section 2 contains two theorems. In the first one we show, in particular, that the local square integrability of $1 / r$ and the fact that

$$
\frac{1}{r} \rightarrow 0 \text { as } r \rightarrow \infty \text {, }
$$

imply that this potential is compact with reference to $\Delta$. Theorem 2.1 is our basic theorem inasmuch as it is used to establish the ones that follow. Theorem 2.2 shows that the resolvent congruence pro- 
perty holds for a wider class of potentials. In $\S 3$ we consider potentials which are bounded from below and Theorem 3.1 formulates conditions under which the Friedrichs extensions of the perturbed and unperturbed operator are resolvent congruent. In $\S 4$ we combine Theorem 2.1 with the coerciveness estimates for elliptic operators with variable coefficients. Specifically in Theorem 4.1 we take the unperturbed operator to be a second order differential operator with variable coefficients acting in free space. The main requirement on the coefficients being uniform ellipticity, including the point at infinity. Then according to this theorem the basic Theorem 2.1 remains valid for such unperturbed operators. From this fact it is easy to derive that to such a perturbed operator there is an appropriate elliptic operator with constant coefficients to which it is resolvent congruent. Hence the essential spectrum of such a perturbed operator is the positive axis. This is the statement of Theorem 4.2. Finally in $\S 5$ we illustrate that similar statements hold for operators acting in the exterior of a bounded region.

It is a pleasure to thank Professors Balslev and Schechter for valuable correspondence and conversations respectively. In particular the author appreciates a Balslev remark, which led to a sharper formulation of the basic Theorem 2.1.

1. The notion of the essential spectrum and Weyl's theorem. The notion of the essential spectrum of an operator can be defined in several different ways [12] [44, Definition 1.19 36, §133]. In a forthcoming work M. Schechter [37] introduces a new such definition, for operators acting on a Banach space, and discusses its relation to these ones. At the same time he introduces criteria on a perturbation, which ensure that it produces no new essential spectrum.

For normal, in particular, self adjoint operators, these definitions are equivalent to the following [39, $§ 2$, Corollary 3]: the essential spectrum of $N, \sigma_{e}(N)$, consists of those points of the spectrum of $N$ which are not isolated point eigenvalues of finite multiplicity. According to Weyl's well known theorem [36, Theorem in $\S 134$ ] if $A$ and $B$ are strictly self adjoint operators and $B-A$ is compact then

$$
\sigma_{e}(A)=\sigma_{e}(B) \text {. }
$$

This theorem was extended to normal operators by J. Schwartz [36, $\S 2$, Corollary 3], who showed that if $N$ and $M$ are normal and $M-N$ is compact then

$$
\sigma_{e}(N)=\sigma_{e}(M)
$$

Suppose that $A$ and $B$ are strictly self adjoint operators on possibly 
different domains and the intersection of their resolvent sets contains a point $\zeta$ such that:

$$
(\zeta-B)^{-1}-(\zeta-A)^{-1} \text { is compact. }
$$

Then setting

$$
M=(\zeta-A)^{-1}
$$

and

$$
N=(\zeta-\beta)^{-1}
$$

in relation (1.2), we see from the spectral mapping theorem that

$$
\sigma_{e}(A)=\sigma_{e}(B) \text {. }
$$

Thus in order to establish that the essential spectra of $A$ and $B$ are equal it suffices to establish relation (1.3). Note that if $A$ is not strictly self adjoint, $(\zeta-A)^{-1}$ may not exist, or it may not be normal. Hence keeping the previously mentioned definition of the essential spectrum this conclusion may not hold. Actually this definition of the essential spectrum has no intrinsic value. We use it for convenience, since at the preparation of this work, the announcement of Schechter [31] was not available.

For brevity we shall refer to relation (1.3) by saying that the operators $A$ and $B$ are $\zeta$-congruent to each other. Following Calkin [16], who initiated the calculus modulo compact operators, in this case we set

$$
(\zeta-A)^{-1}-(\zeta-B)^{-1} \equiv 0
$$

or in short

$$
A \equiv B(\zeta)
$$

Observe that the validity of this relation does not imply that the difference $B-A$ is defined. For, unbounded operators are defined on dense sets and the intersection of two dense sets may be empty.

It is an interesting special case of relation (1.4) in which this difference is defined. To emphasize this fact we set

$$
A=A_{0}, B=A_{1}, A_{1}-A_{0}=P,
$$

and call $P$ the perturbation. Actually instead of relation (1.4) we shall assume that there is a value $\zeta$ in $\rho\left(A_{0}\right)$, the unperturbed resolvent set, such that

$$
P R_{0}(\zeta) \equiv 0, \quad R_{0}(\zeta)=\left(\zeta-A_{0}\right)^{-1}
$$


More specifically we assume that $P R_{0}(\zeta)$ is an everywhere defined operator, for which this holds. Hence we also assume that

$$
\mathfrak{D}\left(A_{0}\right) \subset \mathfrak{D}(P) \text {. }
$$

Note that if this holds for any complex number $\zeta$ in $\rho\left(A_{0}\right)$, then it holds for every $\zeta$ in $\rho\left(A_{0}\right)$. According to $F$. Wolf [44, Corollary to statement 1.11] this property is equivalent to the property introduced by Gokhberg and Krein [24] that $P$ is $A_{0}$-compact.

This property is defined with reference to an operator $A_{0}$ whose resolvent set is not empty, in particular, which is closed on its domain. It is convenient to extend this definition by admitting a closable $A_{0}$. Let $A_{0}$ be closable on some set $\mathfrak{D}$ and as usual, let $\bar{A}_{0}$ be its closure. For convenience, let $P$ on $(D)$ be closable. Suppose that there is a number $\zeta$ in $\rho\left(A_{0}\right)$, such that the closure of $P R_{0}(\zeta)$ on $\left(\zeta-A_{0}\right) \mathfrak{D}$ is compact. Then we claim that $\bar{P}$, the closure of $P$ is $\bar{A}_{0}$-compact. To see this, clearly it suffices to show that

$$
\mathfrak{D}\left(\bar{A}_{0}\right) \subset \mathfrak{D}(\bar{P}) \text {. }
$$

Accordingly let $f$ be in $\mathfrak{D}\left(\bar{A}_{0}\right)$. By assumption $\zeta$ is in $\rho\left(A_{0}\right)$ and hence there is a vector $g$ such that

$$
f=R_{0}(\zeta) g \text {. }
$$

At the same time it follows that the set $\left(\zeta-A_{0}\right) D$ is dense. Hence there is a sequence of vectors $\left\{g_{n}\right\}$ in this set, which tends to $g$. Then setting

$$
f_{n}=R_{0}(\zeta) g_{n},
$$

we obtain a sequence in $\mathscr{D}$, which tends to $f$, and it is clear that for an appropriate subsequence $\left\{P f_{n}\right\}$ is a Cauchy sequence. Thus (1.7) follows and the claim is established. Since every nonreal number belongs to the resolvent set of a strictly self adjoint operator [19, Theorem X. 4.2], such a situation arises if $A_{0}$ and $P$ are essentially and formally self adjoint on some set $\mathfrak{D}$, and $P R_{0}(\zeta)$ is compact for a nonreal $\zeta$. Then the claim says, that it is no loss of generality to assume that $A_{0}$ is strictly self adjoint.

Next we maintain that if $A_{0}$ and $P$ are self adjoint and $P$ is $A_{0}$ compact then under general circumstances $A_{0}+P$ has a unique strictly self adjoint extension, which is resolvent congruent to $A_{0}$. To see this we formulate a lemma, which summarizes some facts, used sometimes implicitly, by Kato [29]. In it, for brevity, we say that an operator is invertible if it admits an everywhere defined and bounded inverse. 
Lemma 1.1. Let the operators $A_{0}$ and $P$ be defined on some dense set $D$ and suppose that $\zeta-A_{0}$ on $\mathfrak{D}$ is invertible. Then a necessary and sufficient condition that $\zeta-A_{1}=\zeta-A_{0}-P$ on $\mathfrak{D}$ be also invertible is that $P$ is $A_{0}$-bounded and $1-P R_{0}(\zeta)$ is invertible. Denoting the inverses by $R_{0}(\zeta)$ and $R_{1}(\zeta)$ we have

$$
R_{1}(\zeta)=R_{0}(\zeta)\left(1-P R_{0}(\zeta)\right)^{-1} \text {. }
$$

Furthermore, if $A_{0}$ and $P$ are strictly and formally self adjoint on (D) respectively and $P$ is $A_{0}$-compact then (1.8) holds for every nonreal $\zeta$. In particular $A_{1}$ is strictly self adjoint on D.

We derive this lemma from the relation

$$
\left(\zeta-A_{1}\right)=\left(1-P R_{0}(\zeta)\right)\left(\zeta-A_{0}\right) \text { on } \mathfrak{D},
$$

which holds since $P$ is $A_{0}$-bounded.

To see the sufficiency of this condition assume that $P R_{0}(\zeta)$ is bounded and $1-P R_{0}(\zeta)$ is invertible. Then we see from this relation that

$$
R_{0}(\zeta)\left(1-P R_{0}(\zeta)\right)^{-1}\left(\zeta-A_{1}\right)=1 \text { on } \mathfrak{D}
$$

and

$$
\left(\zeta-A_{1}\right) R_{0}(\zeta)\left(1-P R_{0}(\zeta)\right)^{-1}=1 \text { on } \mathfrak{S},
$$

if we remember that $R_{0}(\zeta)$ maps $\mathfrak{S}$ onto $\mathfrak{D}$. Thus conclusion (1.8) follows.

To see the necessity of this condition assume that $\zeta-\mathrm{A}_{1}$, on $\mathfrak{D}$ is invertible, and recall that $\zeta-A_{0}$ on $\mathfrak{D}$ is also invertible. Hence the operators $\zeta-A_{1}$, and $\zeta-A_{0}$ are closed on $\mathfrak{D}$. This in general does not imply that $P$, their difference is also closed. Nevertheless we have a weaker property, to which Schechter [37] referred as $P$ being $A$-closed. An elementary argument shows that this weaker property implies that $P R_{0}(\zeta)$ is closed. Clearly this operator is defined on the entire space and hence according to the closed graph theorem [19, Th. II. 2.4] it is bounded. This yields one of the conclusions. By assumption $\zeta-A_{1}$ is invertible on $\mathscr{D}$, in particular it is one to one. We claim that this implies that $1-P R_{0}(\zeta)$ is one to one on the entire space $\mathfrak{S}$. To see this let

$$
\left(1-P R_{0}(\zeta)\right) f=0
$$

1 To emphasize the difference between operators which are Hermitian symmetric and self adjoint in the strict mathematical sense, we speak about formally and strictly self adjoint operators, respectively. 
Since $\zeta-A_{0}$ on $\mathfrak{D}$ is invertible, there is a vector $g$ in $\mathfrak{D}$, such that

$$
f=\left(\zeta-A_{0}\right) g \text {. }
$$

Insertion of these facts in (1.9) yields

$$
\left(\zeta-A_{1}\right) g=0 \text {. }
$$

Since $\left(\zeta-A_{1}\right)$ is one to one on $\mathfrak{D}, g=0$ and $f=0$ follows. Hence $1-$ $P R_{0}(\zeta)$ is one to one on $\mathfrak{S}$. By assumption $\zeta-A_{1}$ maps $\mathfrak{D}$ onto all of $\mathfrak{S}$. Insertion of this fact in (1.9) shows that the range of $1-P R_{0}(\zeta)$ equals $\mathfrak{S}$. Thus this operator on $\mathfrak{S}$ is one to one and onto. Therefore according to a theorem of Banach [19, Th. II. 2.2] it admits a bounded inverse.

Finally we consider the self adjoint case. Then clearly for any nonreal $\zeta, \zeta-A_{1}$ is one to one on D. We have seen in the proof of the necessity part that this implies that $1-P R_{0}(\zeta)$ is one to one on all of $\mathfrak{K}$. Note that since $A_{0}$ is strictly self adjoint and $\zeta$ is nonreal, $R_{0}(\zeta)$ exists. By assumption $P R_{0}(\zeta)$ is compact and thus we can conclude from the Fredholm alternative $\left[19\right.$, p. 609] that $1-P R_{0}(\zeta)$ is invertible. Therefore remembering the already established sufficiency part we see that $\zeta-A$ on $\mathfrak{D}$ is invertible. Since this holds for any nonreal $\zeta$ the strict self adjointness of $A_{1}$ on $\mathfrak{D}$ follows [19, Corollary XII. $4.13(b)$ ].

Having established Lemma 1.1 as a simple consequence, we derive the following:

Proposition. Suppose that $A_{0}$ and $P$ are strictly and formally self adjoint on $D$ respectively, and suppose that $P$ is $A_{0}$ compact. Then $A_{1}=A_{0}+P$ is strictly self adjoint on $\mathfrak{D}$ and it is resolvent congruent to $A_{0}$.

The first conclusion which says that $A_{1}$ is strictly self adjoint on D), the domain of $A_{0}$, appears explicitly in Lemma 1.1. Hence for any nonreal $\zeta$ the operator $\left(\zeta-A_{1}\right) R_{0}(\zeta)$ is defined on all of $\mathfrak{S}$. Then we see from equation (1.9) which appears in the proof of Lemma 1.1 that

$$
\left(\zeta-A_{1}\right) R_{0}(\zeta)=1-P R_{0}(\zeta)
$$

Thus

$$
\left(\zeta-A_{1}\right)\left(R_{1}(\zeta)-R_{0}(\zeta)\right)=P R_{0}(\zeta) .
$$

Since $P R_{0}(\zeta)$ is bounded on all of $\mathfrak{S}$, we obtain 


$$
R_{1}(\zeta)-R_{0}(\zeta)=R_{1}(\zeta) P R_{0}(\zeta)
$$

This is the second resolvent equation and note that since $P$ is possibly unbounded its validity is not obvious.

By assumption $P R_{0}(\zeta)$ is compact and insertion of this fact in (1.10) shows that this difference is compact. Thus $A_{1}$ and $A_{0}$ are resolvent congruent, and this establishes the proposition.

2. Perturbation of the Laplacian in free space by a potential. Let $\dot{\mathfrak{S}}_{2}$ denote the set of twice continuously differentiable functions with bounded support in $\mathscr{E}_{n}$, the $n$-dimensional real Euclidean space. Then as is well known, $-\Delta$, the negative Laplacian, is essentially self adjoint on $\dot{\mathfrak{r}}_{2}$. We denote its closure by $-\Delta$ again, which we take to be the unperturbed operator and accordingly set

$$
R_{0}(\zeta)=(\zeta+\Delta)^{-1}
$$

whenever a bounded inverse exists. Let $p$ be a measurable, locally square integrable real function and assign to it the operator

$$
M(p) f(x)=p(x) f(x),
$$

which is also essentially self adjoint on $\dot{\mathfrak{夭}}_{2}$. In this section we formulate conditions on the function $p$ which ensure that the operator $M(p)$ is A-compact. According to Lemma 1.1, these conditions also ensure that the perturbed operator $-\Delta+M(p)$ is essentially self adjoint on $\dot{\mathfrak{s}}_{2}$, and we set

$$
R_{1}(\zeta)=(\zeta+\Delta-M(p))^{-1} .
$$

The question of the essential self adjointness of this operator was investigated by several authors. Specifically T. Kato [29] formulated conditions on the potential $p$ which ensured that this is the case. His results were extended by Stummel [41], which in turn was extended by Ikebe and Kato [31]. Recently this problem was taken up again by Jörgens [28]. Of course the conditions of these authors for essential self adjointness is much weaker than our conditions, to be stated, for relative compactness.

We start by introducing an expression which is defined with the aid of a weighted integral mean for powers of the potential:

Definition. Given two real numbers $\alpha$ and $\mu$, and a measurable function $p$. Then set

$$
I_{\mu, \alpha}(p)=\sup _{x} \int_{|x-y|<1}|p(y)|^{\mu}|x-y|^{\alpha} d y .
$$


Such an expression was used by Stummel [41, Satz 4.2 and condition (4.4)] to formulate a condition on $p$ under which the operator $-\Delta+M(p)$ is essentially self adjoint on $\dot{\varepsilon}_{2}$. It reads as follows:

Condition $S(\alpha)$. In case of dimensions $n=1,2,3, I_{2,0}(p)<\infty$, and in case of dimensions $n=4,5, \cdots$, to the function $p$, there is a number $\alpha$, such that

$$
\alpha<4-n \text { and } I_{2, \alpha}(p)<\infty .
$$

An overlapping condition was used by Birman [9, Condition $K$ and Condition $R$, p. 142] to ensure that the Friedrichs extensions of the perturbed and unperturbed operators are resolvent congruent. Specifically he showed that this is the case if $I_{1}, \alpha(p)<\infty$, where $\alpha<1-n$. A related condition was used by Brownell [13, Th. 1] for essential self adjointness. His condition requires that to the function $p$ there is a number $\mu$ such that

$$
\mu>\max \left(2, \frac{n}{2}\right) \text { and } I_{\mu, 0}(p)<\infty .
$$

This implies Condition $S(\alpha)$, which is seen by an elementary application of the Hölder inequality [35, Appendix 1].

After these preparations we show that if the function $p$ satisfies Condition $S(\alpha)$ and in addition it is "small" at infinity, then the operator $M(p)$ is $\Delta$-compact. Our first smallness condition is formulated as follows:

Subtheorem 2.1. Suppose that the function p satisfies Condition $S(\alpha)$ and in addition assume that it is square integrable over the entire space. Then the operator $M(p)$ is $\Delta$-compact i.e.

$$
M(p) R_{0}(i) \equiv 0 \text {. }
$$

Here, for the complex number $\zeta$ entering the definition of relative compactness we have taken $\zeta=i$. Actually, according to a result of Wienholtz [43] the Stummel condition ensures that $-\Delta+M(p)$ is bounded from below and hence for $\zeta$, we could have taken a sufficiently small negative number. Nevertheless, we prefer not to make use of the semiboundedness of this operator.

(a) The special cases of dimensions $n=1,2,3$. Since $\triangle$ commutes with translation so does $R_{0}(i)$ and hence it can be represented as convolution by a tempered distribution [25]. We shall need, however, detailed information on this distribution. Specifically, that it 
corresponds to a function $g$, the Green's function, and in view of $n \leqq 3, g$ is square integrable. That is, denoting by $R_{0}(i)(x, y)$ the value of the kernel of $R_{0}(i)$ at the point $(x, y)$, we have

$$
R_{0}(i)(x, y)=g(x-y)
$$

and

$$
\|g\|^{2}=\int|g(u)|^{2} d u<\infty .
$$

In view of our additional assumption, these formulae imply that $\left\|M(p)(i+\Delta)^{-1}\right\|_{2}$, the Hilbert-Schmidt norm of this operator, is finite. For, evidently,

$$
\left\|M(p) R_{0}(i)\right\|_{2}=\left(\iint p^{2}(x) g^{2}(x-y) d y d x\right)^{1 / 2} .
$$

According to (2.3) for each fixed value of the vector $x$

$$
\int p^{2}(x) g^{2}(x-y) d y=p^{2}(x)\|g\|^{2},
$$

which inserted in (2.4) yields

$$
\left\|M(p) R_{0}(i)\right\|_{2}=\|p\|\|g\| \text {. }
$$

Since by assumption $p$ is a square integrable over all of $\mathscr{E}_{n}$ the right member is finite. Hence the Hilbert-Schmidt norm of this operator is finite and conclusion (2.2) follows. In case of dimension $n=1$, this result was obtained by Agudo and Wolf [1]. In case of dimension $n=3$, this generalizes another result of Agudo-Wolf [2], inasmuch as it is not required that $p$ be bounded.

(b) The general case. In spite of the assumption, that $p$ is square integrable over all of $\mathscr{E}_{n}$, it is no longer true that the operator in (2.2) is Hilbert-Schmidt; the reason being that $g$, the Green's function, is not square integrable over all of $\mathscr{E}_{n}$. Nevertheless we have the following estimates [27, Chapter III], [17, Subsection III. 2.2],

$$
g(u)= \begin{cases}0(1) & \text { for } n=1 \\ 0(\log |u|) \text { at }|u|=0, & \text { for } n=2 \\ 0\left(|u|^{2-n}\right) & \text { for } n=3,4, \cdots\end{cases}
$$

and

$$
g(u)=0\left(e^{-\gamma|u|}\right) \text { at }|u|=\infty, \quad \text { for } n=1,2, \cdots .
$$

Here $\gamma$ is an appropriate positive constant. Evidently these estimates 
imply that

$$
\int|g(u)| d u<\infty,
$$

moreover, for every positive number $\delta$

$$
\int_{|u|>\delta}|g(u)|^{2} d u<\infty .
$$

Next set

$$
g_{\delta}(u)=\left\{\begin{array}{ll}
g(u) & |u|>\delta \\
0 & |u|<\delta
\end{array},\right.
$$

and

$$
R_{0, \delta}(i)(x, y)=g_{\delta}(x-y) .
$$

Then, from the assumption that $p$ is square integrable over all of $\mathscr{E}_{n}$, we see that

$$
M(p) R_{0, \delta}(i) \equiv 0,
$$

since $R_{0, \delta}(i)$ is convolution by the function $g_{\delta}$ which in view of $(2.6)_{\delta}$ is square integrable over the entire space.

Relation (2.7) shows that in order to establish conclusion (2.2), it suffices to establish

$$
\lim _{\delta \rightarrow 0}\left\|M(p) R_{0}(i)-M(p) R_{0, \delta}(i)\right\|=0 .
$$

To see the validity of this relation, we need an observation of Friedrichs [20, relations (20.07) and (20.08)], which gives a generalization of the Hilbert-Schmidt bound. Specifically for an arbitrary integral operator $K$ and for the given number $\beta, 0 \leqq \beta \leqq 1$, he introduced

$$
\|K\|_{2 \beta}=\sup _{z} \iint|K(x, y)|^{2 \beta}|K(x, z)|^{2-2 \beta} d y d x
$$

and showed that

$$
\|K\| \leqq\|K\|_{2 \beta} \text {. }
$$

We apply this bound to the operator

$$
A_{\delta}=M(p) R_{0}(i)-M(p) R_{0, \delta}(i)
$$

which evidently has the kernel

$$
A_{\delta}(x, y)=\left\{\begin{array}{ll}
p(x) g(x-y) & |x-y|<\delta \\
0 & |x-y|>\delta
\end{array} .\right.
$$


Hence

(2.11) $\quad\left\|A_{\delta}\right\|_{2 \beta} \leqq \sup _{z} \iint_{|x-z|<\delta} p^{2}(x)|g(x-y)|^{2 \beta}|g(x-z)|^{2-2 \beta} d y d x$.

First we choose the value $2 \beta$ in such a way that

$$
0 \leqq 2 \beta \leqq 2, \quad \int|g(u)|^{2 \beta} d u<\infty .
$$

In view of estimates (2.5) in case of dimensions $n=1,2,3$ such a value is $2 \beta=2$. At the same time it follows that in case of higher dimensions this relation is implied by

$$
2 \beta(-n+2)>-n, \quad 0 \leqq 2 \beta \leqq 2,
$$

if we remember that, introducing polar coordinates in (2.12), the weight function is a constant multiple of $|u|^{n-1}$. For such values of $2 \beta$ the $y$-integration in (2.11) can be carried out, yielding

$$
\left\|A_{\delta}\right\|_{2 \beta}= \begin{cases}0(1) \sup _{z} \int_{|x-z|<1} p^{2}(x) d x & \text { for } n=1,2,3, \\ 0(1) \sup _{z} \int p^{2}(x)|g(x-z)|^{2-2 \beta} d x & \text { for } n=4,5, \cdots\end{cases}
$$

This in turn, upon insertion of estimate $(2.5)_{0}$, yields

$$
\left\|A_{\delta}\right\|_{2 \beta}= \begin{cases}0(1) \sup _{z} \int_{|x-z|<1} p^{2}(x) d x, & \text { for } n=1,2,3 \\ 0(1) \sup _{z} \int_{|x-z|<1} p^{2}(x)|x-z|^{(2-2 \beta)(2-n)} d x & , \text { for } n=4,5, \cdots\end{cases}
$$

Our first requirement on $2 \beta$ was that it should be small enough to allow the integral in (2.12) to converge. Now we impose the second requirement on $2 \beta$, namely that it should be large enough and hence $2-2 \beta$ should be small enough to make the right member of (2.14) small. Specifically

$$
(2-2 \beta)(2-n)>\alpha^{\prime}
$$

where $\alpha^{\prime}$ is an arbitrary number such that

$$
4-n>\alpha^{\prime}>\alpha,
$$

and $\alpha$ is the value entering Condition $S(\alpha)$. Note that in view this condition there is such a value of $\alpha^{\prime}$. Next we observe the following elementary fact, which we shall not verify: for any $\alpha^{\prime}$ which satisfies the left inequality of (2.16), inequalities (2.13) and (2.15) are compatible, provided that $n>3$, which is the case. Hence choosing $2 \beta$ to satisfy 
both of these inequalities (2.14) yields

$$
\left\|A_{\delta}\right\|_{2 \beta}= \begin{cases}0(1) \sup _{z} \int_{|x-z|<\delta} p^{2}(x) d x & \text { for } n=1,2,3 \\ 0(1) \sup _{z} \int_{|x-z|<\delta} p^{2}(x)|x-z|^{\alpha^{\prime}} d x & \text { for } n=4,5, \cdots\end{cases}
$$

Now it is an interesting fact, which was pointed out to the author by E. Balslev, that in case of $n>3$, Condition $S(\alpha)$ alone, implies

$$
\sup _{z} \int_{|x-z|>\delta} p^{2}(x)|x-z|^{\alpha^{\prime}} d x=o(1) \quad \text { at } \delta=0 \text {. }
$$

This is clear from

$$
\int_{|x-z|<\delta} p^{2}(x)|x-z|^{\alpha^{\prime}} d x<\delta^{\left(\alpha^{\prime}-\alpha\right)} \int_{|x-z|<\delta} p^{2}(x)|x-z|^{\alpha} d x
$$

and from definition (2.16), which show that the exponent of $\delta$ is positive. On the other hand we assumed that $p$ is square integrable over all of $\mathscr{E}_{n}$, hence

$$
\sup _{z} \int_{|x-z|<\delta} p^{2}(x) d x=\mathrm{o}(1) \quad \text { at } \delta=0 .
$$

Insertion of relations (2.19) and (2.18) in relation (2.17) yields

$$
\left\|A_{\delta}\right\|_{2 \beta}=\mathrm{o}(1) \text { at } \delta=0 \text {, }
$$

for all dimensions, $n=1,2, \cdots$, . This in turn, yields the validity of relation (2.8), if we remember definition (2.10) and the Friedrichs bound (2.9). Thus conclusion (2.2) is established and this completes the proof of Subtheorem 2.1. Note that one of the assumptions was that $p$ is square integrable over the entire space and the proof of relation (2.8) used only a weaker assumption. For, in case of dimensions $n=1,2,3$, we needed relation (2.9) and in case of dimensions $n>4$ the sole Condition $S(\alpha)$ was needed.

Clearly this assumption on $p$ cannot be removed completely. For, the function $p(x) \equiv 1$ evidently satisfies Condition $S(\alpha)$ and the operator $M(p)$ is not compact with respect to $-\Delta$, since the spectrum of $-\Delta+M(p)$ consists of $[1, \infty]$. We maintain, however, that this condition can be weakened and to see this we formulate a more general smallness condition at infinity. In it let $\mathscr{S}(x, 1)=\mathscr{S}(x)$ denote the sphere of radius 1 with center at $x$ in $\mathscr{E}_{n}$.

Condition $B(\mu)$. Given the positive number $\mu$,

$$
\lim _{x \rightarrow \infty} \int_{\mathscr{S}(x)}|p(y)|^{\mu} d y=0 \text {. }
$$


This is a one parameter family of conditions labeled by $\mu$, which for the value $\mu=1$ gives the original one of Birman [9, Condition $\mathrm{K}, \mathrm{p} .142]$. Actually this family of sphere conditions was stated for future reference only, since in the theorem that follows we choose another special value, namely $\mu=2$.

THEOREM 2.1. Suppose that the real valued function $p$ satisfies Condition $S(\alpha)$ and Condition $B(2)$. Then the operator $M(p)$ is $\Delta-$ compact i.e.

$$
M(p) R_{0}(i) \equiv 0
$$

Moreover

$$
\sigma_{e}(-\Delta+M(p))=\sigma_{e}(-\Delta)=[0, \infty)
$$

We shall establish this theorem by showing that the operator $M(p) R_{0}(i)$ is the limit in the norm of a sequence of compact operators. Specifically, setting

$$
p_{k}(x)=\left\{\begin{array}{ll}
p(x) & |x|<k \\
0 & |x|>k
\end{array},\right.
$$

we maintain that

$$
\lim _{k \rightarrow \infty} \lim _{\delta \rightarrow 0}\left\|M(p) R_{0}(i)-M\left(p_{k}\right) R_{0, \delta}(i)\right\|=0 .
$$

Since the function $p_{k}$ is square integrable over the entire space, relation (2.7) appearing in the proof of Subtheorem 2.1 applies to it and hence each of the operators $M\left(p_{k}\right) R_{0, \delta}(i)$ is compact.

To see the validity of relation $(2.20)$ we first show that,

$$
\lim _{k \rightarrow \infty}\left\|M(p) R_{0, \delta}(i)-M\left(p_{k}\right) R_{0, \delta}(i)\right\|=0,
$$

$\delta$ being arbitrary but fixed. Setting

$$
B_{k}=M(p) R_{0, \delta}(i)-M\left(p_{k}\right) R_{0, \delta}(i),
$$

we evidently have

$$
\left\|B_{k}\right\|_{1}=\sup _{z} \iint\left|p(x)-p_{k}(x)\right|^{2}|g(x-y)||g(x-z)| d y d x,
$$

the integral being extended over $|x-y|>\delta$ and $|x-z|>\delta$. Hence carrying out the $y$-integration yields

$$
\left\|B_{k}\right\|_{1}=0(1) \sup _{z} \int_{|x-z|>\delta}\left|p(x)-p_{k}(x)\right|^{2} e^{-\gamma|x-z|} d x,
$$

if we remember estimate $(2.5)_{\infty}$. Now clearly 


$$
\int e^{-\gamma|x-z|}\left|p(x)-p_{k}(x)\right|^{2} d x=\sum_{m=0}^{\infty} e^{-\gamma m} \alpha(z, k, m)
$$

where

$$
\alpha(z, k, m)=\int_{m<|x-z|<m+1}\left|p(x)-p_{k}(x)\right|^{2} d x
$$

Next consider the sequence of numbers

$$
\frac{\alpha(z, k, m)}{m^{n-1}}
$$

$n$ being the dimension of the space $\mathscr{E}_{n}$ over which our operators are defined. We claim the assumption that the potential $p$ satisfies Condition $B(2)$ implies that these numbers tend to zero, as $k$ tends to infinity, and that this is uniform in $z$ and $m$. To see this we reformulate the Birman sphere condition with the aid of cubes of unit length. Then cover the spherical shell, $m<|x-z|<m+1$, in the $x$-space by unit cubes in such a way that each point is either covered exactly once, or is on the boundary of several cubes. This way actually we cover a set which is slightly larger than the shell. Nevertheless since the volume of the shell is proportional to $m^{n-1}$ we see the validity of the claim. This shows that for an arbitrary positive number $\gamma$,

$$
\lim _{k \rightarrow \infty}\left[\sup _{z} \int e^{-\gamma|x-z|}\left|p(x)-p_{k}(x)\right|^{2} d x\right]=0 \text {. }
$$

Insertion of this relation in (2.23) yields

$$
\lim _{k \rightarrow \infty}\left\|B_{k}\right\|_{1}=0 \text {. }
$$

Thus remembering definition (2.22) and the Friedrichs bound (2.9), we see the validity of relation (2.21).

Since Condition $B(2)$ and Condition $S(\alpha)$ clearly imply relation (2.19), relation (2.8) entering the proof of Subtheorem 2.1, holds under the present assumptions. Finally combining relations (2.8) and (2.21) we arrive at the validity of relation (2.20). Thus, the operator $M(p) R_{0}(i)$ is compact, and this completes the proof of Theorem 2.1.

Clearly, the Coulomb potential $-1 / r$ satisfies the assumptions of Theorem 2.1, which show that the essential spectrum of the hydrogen energy operator consists of the positive axis, i.e.

$$
\sigma_{e}\left(-\Delta-\frac{1}{r}\right)=[0, \infty) \text {. }
$$

Having established Theorem 2.1 let us return to Condition $B(\mu)$ for arbitrary positive $\mu$. Note that an elementary application of 
Hölder's inequality shows that for $\mu_{1} \leqq \mu_{2}$, Condition $B\left(\mu_{2}\right)$ implies Condition $B\left(\mu_{1}\right)$, the converse implication being false in general. Hence only the case $0<\mu \leqq 2$ is of interest. We do not claim that replacing Condition $B(2)$ by such a Condition $B(\mu)$ the conclusion of Theorem 2.1 still holds. In fact the remark after the following Theorem 2.2 shows that in general this is false. Nevertheless we have the slightly weaker conclusion that the perturbed and unperturbed operators are resolvent congruent. This is the statement of the theorem that follows.

THEOREM 2.2. Suppose that the real valued function $p$ satisfies Condition $S(\alpha)$ and Condition $B(\mu), \mu$ being arbitrary and positive. Then the operator $-\Delta+M(P)$ is essentially self adjoint on $\dot{\mathfrak{F}}_{2}$. Furthermore it is resolvent congruent to -4 , i.e.

$$
R_{1}(i)-R_{0}(i) \equiv 0,
$$

and

$$
\sigma_{e}(-\Delta+M(p))=[0,+\infty) .
$$

According to a result of Kato [29] and Stummel [41], Condition $S(\alpha)$ alone implies that

$$
\lim _{|\rho| \rightarrow \infty}\left\|M(p) R_{0}(i \rho)\right\|=0 .
$$

This relation could also be derived [35, Appendix II], using a variant of relation (2.24), but we shall not be concerned with this fact. We choose a fixed value of $\rho$ in such a way that

$$
\left\|M(p) R_{0}(i \rho)\right\|<1 \text {. }
$$

Then clearly the operator $1-M(p) R_{0}(i \rho)$ admits an everywhere defined bounded inverse. Hence according to Lemma 1.1 the perturbed operator is essentially self adjoint on $\dot{\mathfrak{F}}_{2}$. At the same time it follows from equation (1.8) that

$$
R_{1}(i \rho)-R_{0}(i \rho)=R_{0}(i \rho) M(p) R_{0}(i \rho) \cdot\left(1-M(p) R_{0}(i \rho)\right)^{-1},
$$

if we remember that for arbitrary $A$

$$
(1-A)^{-1}-1=A(1-A)^{-1} \text {. }
$$

We claim that the first factor is compact. To see this set

$$
\begin{aligned}
s(x) & =\operatorname{sgn} p(x) \\
p_{1}(x) & =|p(x)|^{1-(\mu / 2)} \\
p_{2}(x) & =|p(x)|^{\mu / 2} .
\end{aligned}
$$


Then clearly, the first factor in turn, can be factored as

$$
R_{0}(i \rho) M\left(p_{1}\right) \cdot M(s) \cdot M\left(p_{2}\right) R_{0}(i \rho) .
$$

Since $0<\mu \leqq 2$, definition (2.29) shows that

$$
p_{1}^{2} \leqq 1+p^{2} \quad \text { and } \quad p_{2}^{2} \leqq 1+p^{2} .
$$

Combining this fact with the assumption that the function $p$ satisfies Condition $S(\alpha)$, we see that the function $p_{1}$ and $p_{2}$ do too. On the other hand from the assumption that $p$ satisfies Condition $B(\mu)$ we see that $p_{2}$ satisfies Condition $B(2)$. Hence according to Theorem 2.1 the third factor in (2.30) is compact. Next recall that an estimate similar to $(2.27)$ shows that

$$
\text { \| } M\left(p_{1}\right) R_{0}(-i \rho) \|<\infty .
$$

Since this operator on $(i \rho+\Delta) D$ is formal adjoint to $R_{0}(i \rho) M\left(p_{1}\right)$ on D, the latter operator is bounded. Thus the operator in $(2.30)$ is compact, which inserted in (2.28) yields the validity of conclusion (2.26) and Theorem 2.2 is established.

Theorem 2.2 is a family of theorems labeled by $\mu$. For the value $\mu=2$ the hypothesis coincide with the ones of Theorem 2.1. We observe that replacing this value of $\mu$ by a strictly smaller one, $0<\mu<2$, the resulting hypothesis are strictly weaker. For, it was noted before that in this case Condition $B(2)$ implies Condition $B(\mu)$. Also, it is easy to construct a potential which satisfies Condition $B(\mu)$ and Condition $S(\alpha)$ but does not satisfy Condition $B(2)$. It is an interesting fact, however, observed by W. Roelcke that there is no best possible choice of $\mu$. For, an elementary argument, which involves the Hölder inequality, shows the following: if the function $p$ satisfies Condition $S(\alpha)$ and Condition $B\left(\mu_{1}\right)$ then it also satisfies Condition $B\left(\mu_{2}\right)$, provided that $\mu_{2}$ is strictly less than 2 .

Finally let us observe that in order that the relation

$$
M\left(p^{\mu}\right) R_{0}(i) \equiv 0
$$

should hold, the validity of Condition $\beta(2 \mu)$ is necessary. This observation is an extension of an earlier one of Molchanov [34]. To see it we proceed indirectly and assume accordingly that the potential does not satisfy this condition. Then there is a sequence of spheres, $\left\{\mathscr{S}\left(x_{k}\right)\right\}$, such that even the spheres $\left\{\mathscr{S}\left(x_{k}, 2\right)\right\}$ are disjoint from each other, and

$$
\lim _{x_{k} \rightarrow \infty} \int_{\mathscr{S}\left(x_{k}\right)}|p(x)|^{2 \mu} d x>0 .
$$

Let $j(x)$ be a smooth function of the variable $x$ such that its support is in $\mathscr{S}(0,2)$ and 


$$
j(x) \geqq 1, \text { for } x \text { in } \mathscr{S}(0,1) .
$$

We maintain that setting

$$
T\left(x_{k}\right) f(x)=f\left(x-x_{k}\right)
$$

and

$$
g_{k}=(i+\Delta) T\left(x_{k}\right) j,
$$

we obtain a bounded sequence which is not mapped into a compact sequence by $M\left(p^{\mu}\right) R_{0}(i)$. To see this first note that $\left\{T\left(x_{k}\right)\right\}$ is a family of isometries which commute with $\Delta$, hence

$$
\left\|(i+\Delta) T\left(x_{k}\right) j\right\| \leqq\|j\|+\|\Delta j\| \text {. }
$$

Next recall that by assumption for $k \neq 1$ the spheres $\mathscr{S}\left(x_{k}, 2\right)$ and $\mathscr{S}\left(x_{1}, 2\right)$ are disjoint, which shows that the support of the functions $T\left(x_{k}\right) j$ and $T\left(x_{1}\right) j$ are also disjoint. Hence

$$
\begin{aligned}
& \left\|M\left(p^{\mu}\right) R_{0}(i) g_{k}-M\left(p^{\mu}\right) R_{0}(i) g_{1}\right\|^{2} \\
& \quad=\left\|M\left(p^{\mu}\right) T\left(x_{k}\right) j-M\left(p^{\mu}\right) T\left(x_{1}\right)\right\|^{2} \\
& \quad=\left\|M\left(p^{\mu}\right) T\left(x_{k}\right) j\right\|^{2}+\left\|M\left(p^{\mu}\right) T\left(x_{1}\right) j\right\|^{2} .
\end{aligned}
$$

Insertion of inequality (2.31) in this relation yields

$$
\left\|M\left(p^{\mu}\right) R_{0}(i) g_{k}-M\left(p^{\mu}\right) R_{0}(i) g_{\ell}\right\|_{2} \geqq \int_{\mathscr{S}\left(x_{k}\right)}|p(x)|^{2 \mu} d x+\int_{\mathscr{S}\left(x_{1}\right)}|p(x)|^{2 \mu} d x .
$$

Thus we see from relation (2.31) that the sequence

$$
\left\{M\left(p^{\mu}\right) R_{0}(i) g_{k}\right\}
$$

is not compact.

3. Potentials which are bounded from below. Recall that Theorem 2.2 was established using an appropriate factorization of the difference of the perturbed and unperturbed resolvents. This in turn, was made possible by an appropriate factorization of the potential. Clearly, this is not unique and for different classes of potentials different factorizations are suitable. Such a factorization is the essential feature of the abstract theorem of Birman [9, Th. 1.2] of our Theorem 2.2 and of the theorem to be stated in this section.

We consider potentials which are bounded from below and for brevity assume that they are positive. In the theorem that follows we formulate a factorization for such potentials, which ensures that the Friedrichs extension [19, Section XII. 5] [20, Th. 31.1] of the perturbed and unperturbed operators are resolvent. For this purpose it is convenient to express the perturbed resolvent in terms of the 
unperturbed resolvent and the perturbation. This is done in the lemma that follows, in which the subscript $F$ denotes the Friedrichs extension of the operator. Note that since the perturbed operator need not be essentially self adjoint on the domain of the unperturbed operator, equation (1.8) of Lemma 1.1 need not hold.

Lemma 3.1. Suppose that the operators $A$ and $P$ are formally self adjoint on the dense set $\mathfrak{D}$ and

$$
(f, A f) \geqq(f, f), \quad(f, P f) \geqq 0, \quad \text { on } \mathfrak{D} .
$$

Then the set $A_{F}^{112} \mathfrak{D}$ is also dense and

$$
\left(g,\left(1+A_{F}^{-1 / 2} P A_{F}^{-1 / 2}\right) g\right) \geqq(g, g) \text { on } A_{F}^{1 / 2} \mathfrak{D} \text {. }
$$

Furthermore

$$
(A+P)_{F}^{-1}=A_{F}^{-1 / 2}\left(1+A_{F}^{-1 / 2} P A_{F}^{-1 / 2}\right)^{-1} A_{F}^{-1 / 2} .
$$

The conclusion that $A_{F}^{1 / 2} D$ is dense is implicit in the Friedrichs construction and was emphasized by Kato [30, §1.6]. Inequality (3.2) is clearly implied by assumption (3.1).

Since $A$ is bounded away from zero,

$$
\mathfrak{D}\left(A_{F}\right) \subset \mathfrak{D}\left(A_{F}^{1 / 2}\right),
$$

and so $A_{F}^{1 / 2}$ is defined on $\mathfrak{D}$. Hence

$$
A+P=A_{F}^{1 / 2}\left(1+A_{F}^{-1 / 2} P A_{F}^{-1 / 2}\right) A_{F}^{1 / 2} \quad \text { on } \mathfrak{D} \text {. }
$$

Next we show that

$$
A_{F^{\prime}}^{1 / 2} \mathfrak{D}(A+P)_{F} \subset \mathfrak{D}\left(1+A_{F}^{-1 / 2} P A_{F}^{-1 / 2}\right)_{F} .
$$

To see this, recall that by definition

$$
\mathfrak{D}(A+P)_{F}=\mathfrak{D}(A+P)^{*} \cap \mathfrak{D}[A+P] .
$$

Here the second symbol on the right, denotes the domain of the closure of the form

$$
[A+P](f, f)=((A+P) f, f) \text { on } \mathfrak{D} \text {. }
$$

We see from inequality (3.2) that

$$
A_{F}^{1 / 2} \mathfrak{D}[A+P]=\mathfrak{D}\left[1+A_{F}^{-1 / 2} P A_{F}^{-1 / 2}\right],
$$

if we remember that $A_{F}^{1 / 2}$ on $\mathcal{D}$ is formally self adjoint and hence closable. We claim that

$$
A_{F}^{1 / 2} \mathfrak{D}(A+P)_{F} \subset \mathfrak{D}\left(1+A_{F}^{-1 / 2} P A_{F}^{-1 / 2}\right)^{*} .
$$


To verify (3.8), for an arbitrary vestor $h$ in $\mathfrak{D}(A+P)_{F}$

$$
h^{*}=(A+P)^{*} h \text {. }
$$

Then for every $f$ in $\mathfrak{D}$ we have,

$$
\left(A_{F}^{1 / 2} h,\left(1+A_{F}^{-1 / 2} P A_{F}^{-1 / 2}\right) A_{F}^{1 / 2} f\right)=(h,(A+P) f)=\left(h^{*}, f\right)=\left(A_{F}^{-1 / 2} h^{*}, A_{F}^{1 / 2} f\right) .
$$

Setting

$$
e^{*}=A_{F}^{-1 / 2} h^{*}, e=A_{F}^{1 / 2} h, g=A_{F}^{1 / 2} f,
$$

in the first and last of these equations yields, for every $g$ in $A_{F}^{1 / 2} \mathscr{D}$

$$
\left(e,\left(1+A_{F}^{-1 / 2} P A_{F}^{-1 / 2}\right) g\right)=\left(e^{*}, g\right) .
$$

Remembering the definition of the adjoint operator, this equation says that $e$ is in the domain of the adjoint of $1+A_{F}^{-1 / 2} P A_{F}^{-1 / 2}$ on $A_{F}^{1 / 2} \mathfrak{D}$. This establishes (3.8), which together with (37) establishes (3.5),

From this relation we derive that the factorization given in equation (3.4) remains valid for the extended operators. To see this let $f \in \mathfrak{D}(A+P)_{F}$. Then remembering that $P$ is positive, the considerations of Friedrichs [20, Th. 31.1] [19, §XII.5] show that there is a sequence $\left\{f_{n}\right\}$ in $\mathfrak{D}$, such that

$$
\begin{gathered}
\lim _{n} A_{F}^{1 / 2} f_{n}=A_{F}^{1 / 2} f \\
\lim _{n} f_{n}=f
\end{gathered}
$$

and for every $h$ in $\mathfrak{D}$

$$
\lim _{n}\left(h,(A+P) f_{n}\right)=(h,(A+P) f) .
$$

We maintain that

$$
\lim _{n}\left(h, A_{F}^{1 / 2}(A+P) f_{n}\right)=\left(h,\left(1+A_{F}^{-1 / 2} P A_{F}^{-1 / 2}\right)_{F} A_{F}^{1 / 2} f\right) .
$$

For, an elementary argument shows that

$$
\left(h,\left(1+A_{F}^{-1 / 2} P A_{F}^{-1 / 2}\right) A_{F}^{1 / 2} f_{n}\right)=\left(\left(1+A_{F}^{-1 / 2} P A_{F}^{-1 / 2}\right) h, A_{F}^{1 / 2} f_{n}\right),
$$

and hence according to $(3.10)_{1}$

$$
\lim _{n}\left(h,\left(1+A_{F}^{-1 / 2} P A_{F}^{-1 / 2}\right) A_{F}^{1 / 2} f_{n}\right)=\left(\left(1+A_{F}^{-1 / 2} P A_{F}^{-1 / 2}\right) h, A_{F}^{1 / 2} f\right) .
$$

Now we make use of the fact that according (3.5) the vector $A_{F}^{1 / 2} f$ is 
in the domain of the extended operator, which is self adjoint. Hence

$$
\left(\left(1+A_{F}^{-1 / 2} P A_{F}^{-1 / 2}\right) h, A_{F}^{1 / 2} f\right)=\left(h,\left(1+A_{F}^{-1 / 2} P A_{F}^{-1 / 2}\right)_{F} A_{F}^{1 / 2} f\right) .
$$

Since on $(D)$ factorization (3.4) holds, the last two equations together imply relation (3.11). Remembering that $h$ was an arbitrary vector in the dense set $\mathfrak{D}$, we see from this relation and from $(3.10)_{3}$ that

$$
A_{F}^{-1 / 2}(A+P)_{F}=\left(1+A_{F}^{-1 / 2} P A_{F}^{-1 / 2}\right)_{F} A_{F}^{1 / 2} \text { on } \mathfrak{D}(A+P)_{F} \text {. }
$$

This equation shows that the operator on the right maps the extended domain $\mathfrak{D}(A+P)_{F}$ into the extended domain $\mathfrak{D}\left(A_{F}^{1 / 2}\right)$. Thus the factorization (3.4) remains valid for the extended operators, that is

$$
(A+P)_{F}=A_{F}^{1 / 2}\left(1+A_{F}^{-1 / 2} P A_{F}^{-1 / 2}\right)_{F} A_{F}^{1 / 2} \quad \text { on } \mathfrak{D}(A+P)_{F} .
$$

Since all three factors on the right are invertible, this implies conclusion (3.3), and completes the proof of Lemma 3.1.

We use this lemma and Theorem 2.1 to formulate a condition on the potential function $p$, which ensures that the Friedrichs extensions of $-\Delta$ and $-\Delta+(M(p))$ are resolvent congruent. Lemma 3.1 plays the same role in the proof of the theorem that follows as Lemma 1.1 did in the proof of Theorem 2.2.

THEOREM 3.1. Suppose that the function $p$ is positive and locally square integrable. Suppose further that it can be factored as

$$
p(x)=p_{1}(x) p_{2}(x),
$$

where $p_{1}$ is such that

$$
\mathrm{I}_{2,2-n}\left(p_{1}\right)<\infty
$$

and $p_{2}$ satisfies Condition $S(\alpha)$ and Condition $B(2)$. Then the value $\zeta=1$ is in the resolvent set of the Friedrichs extensions of $\triangle$ and $\Delta-M(p)$ on $\dot{\mathfrak{C}}_{2}$. Furthermore

$$
(1-\Delta+M(p))_{F}^{-1}-(1-\Delta)_{F}^{-1} \equiv 0 \text {. }
$$

Note that previously the unperturbed operator $A_{0}$, was $-\Delta$ and now its is 4 . This slight inconvenience is due to the fact that we have taken $R_{0}(\zeta)=\left(\zeta-A_{0}\right)^{-1}$ and we wish to make $\zeta-A_{0}$ positive.

As is well known [20, Th. 31.1] [19, §XII. 5] the Friedrichs extension preserves the lower bound of an operator and hence $\zeta=1$ is in the resolvent set of both extended operators.

To see conclusion (3.13) we first claim that there is a dense set $\subseteq$ which is mapped by $(1-\Delta)_{F}^{-1}$ into the domain of $M(p)$. For, the 
set

$$
\mathfrak{S}=(1-\Delta)_{F}^{-1} \dot{\mathfrak{S}}_{2}
$$

is dense, since $1-\Delta$ is essentially self adjoint on $\dot{\mathfrak{S}}_{2}$. Then from the assumption that $p$ is locally square integrable we see the validity of the claim. Therefore the second resolvent equation applies on $\mathfrak{S}$, i.e.,

$$
(1-\Delta+M(p))_{F}^{-1}-(1-\Delta)_{F}^{-1}=(1-\Delta+M(p))_{F}^{-1} M(p)(1-\Delta)_{F}^{-1} .
$$

Note that since the perturbed operator need not be essentially self adjoint on $\dot{\mathfrak{G}}_{2}$ this equation need not hold on the entire space.

Since $1-\Delta$ on $\dot{\mathfrak{F}}_{2}$ is essentially self adjoint, its Friedrichs extension equals its closure. This fact and the assumptions on $p_{2}$ imply, according to Theorem 2.1 that

$$
M\left(p_{2}\right)(1-\Delta)_{F}^{-1} \equiv 0 \text {. }
$$

Finally from Lemma 3.1 we derive that

$$
\left\|(1-\Delta+M(p))_{F}^{-1} M\left(p_{1}\right)\right\|<\infty .
$$

For, according to this lemma

$$
\begin{aligned}
& (1-\Delta+M(p))_{F}^{-1} M\left(p_{1}\right) \\
= & (1-\Delta)_{F}^{-1 / 2} \cdot\left(1+(1-\Delta)^{-1 / 2} M(p)(1-\Delta)^{-1 / 2}\right)_{F}^{-1} \cdot(1-\Delta)_{F}^{-1 / 2} M\left(p_{1}\right)
\end{aligned}
$$

and the first two factors are bounded. We claim that

$$
\| M\left(p_{1}\right)(1-\Delta)^{-1} M\left(p_{1}\right)_{\|}<\infty \text {. }
$$

For, this is an integral operator and its kernel is given by

$$
M\left(p_{1}\right)(1-\Delta)^{-1} M\left(p_{1}\right)(x, y)=p_{1}(x) g(x-y) p_{1}(y) .
$$

On the other hand, from assumption (3.13) we see that

$$
\left.\sup _{x} \int|g(x-y)||| p_{1}(y)\right|^{2} d y<\infty,
$$

if we remember definition (2.1), estimate $(2.5)_{0}$, and the way the expression in (2.23) was estimated. Hence the Carleman norm associated with the weight function $p_{1}(y)$, of the operator in (3.19) is finite. Therefore this operator is bounded [20, relation (20.18)], and (3.19) is established. Since the operators $(1-\Delta)^{-1 / 2} M\left(p_{1}\right)$ and $M\left(p_{1}\right)(1-\Delta)^{-1 / 2}$ are formal adjoints to each other on $\dot{\mathfrak{\mho}}_{2}$, this relation implies that

$$
\left\|(1-\Delta)^{-1 / 2} M\left(p_{1}\right)\right\|<\infty \text {. }
$$

Insertion of this relation in (3.18) yields the validity of (3.17). Then 
insertion of this relation and of (3.16) in (3.15) shows the validity of conclusion (3.14). This completes the proof of Theorem 3.1.

This theorem overlaps with a theorem of Balslev [6], which extends a result of Birman [9].

4. Elliptic operators with variable coefficients. As is well known, every formally self adjoint second order elliptic operator with constant coefficients is unitarily equivalent to the Laplacian, via a change of independent variables [17, Subsection III. 3.1]. Since the validity of Conditions $S(\alpha)$ and $B(2)$ is not affected by such a change of variables, Theorem 2.1 holds for such unperturbed operators.

In this section we define the unperturbed operator with the aid of a formally self adjoint second order differential operator with variable coefficients which has no $0^{\text {th }}$ order term and acts in free space. The main requirement on the coefficients is ellipticity, which is to hold uniformly including the point at infinity. In Theorem 4.1 we combine the coerciveness estimates for elliptic operators [3] [38, Lemma 6] with Theorem 2.1 and show that it remains valid for such unperturbed operators. In other words, if the potential satisfies the conditions of Theorem 2.1 then the Gokhberg-Krein property holds for it. From this fact it is easy to derive, using Theorem 2.1 and the coerciveness estimates again, that such a perturbed operator is resolvent congruent to an appropriate elliptic operator with constant coefficients. Hence its essential spectrum consists of the positive axis. This is the statement of Theorem 4.2.

To be more specific let $L$ be the form

$$
L=\sum_{\substack{j=1 \\ k=1}}^{n} D_{j} M\left(a_{j k}\right) D_{k},
$$

where the Hermitian matrix $\left\{a_{j k}(x)\right\}$ is positive definite at every point $x$. Suppose that these functions are continuously differentiable at any finite point and at infinity we have,

$$
\lim _{|x| \rightarrow \infty} a_{j k}(x)=\alpha_{j k}, \text { and } \lim _{\mid x \rightarrow \infty} \frac{\partial a_{j k}(x)}{\partial x_{i}}=0 .
$$

Suppose further that the matrix $\left\{\alpha_{j k}\right\}$ is positive definite and set

$$
A=\sum_{\substack{j=1 \\ k=1}} M\left(a_{j k}\right) D_{j} D_{k} .
$$

Friedrichs showed [21] that under condition (4.2) the weak and strong extensions of the operator $L$ in $\dot{\mathfrak{S}}_{2}$ are equal, that is the closure equals the strict adjoint. This means that this operator is essentially self adjoint and we denote its closure by $L$ again. After these preparations 
we formulate:

THEOREM 4.1. Suppose that the operator $L$ satisfies condition (4.2) and the function $p$ satisfies the conditions of Theorem 2.1. Then the operator $M(p)$ is L-compact, that is

$$
M(p)(i+L)^{-1} \equiv 0 \text {. }
$$

It is part of conclusion (4.4) that this operator is defined on the entire space $\mathfrak{R}_{2}\left(\mathscr{E}_{n}\right)$, that is

$$
\mathfrak{D}(L) \subset \mathfrak{D}(M(p)) .
$$

It is easily seen from Theorem 2.1 that

$$
\mathfrak{D}(A) \subset \mathfrak{D}(M(p)),
$$

and hence relation (4.5) is implied by

$$
\mathfrak{D}(L) \subset \mathfrak{D}(A) \text {. }
$$

To see the validity of this relation we need the coerciveness estimate of Browder, [12, Th. 2], which holds for more general operators $L$, and says the following: there is a constant $\gamma$ such that for every $f$ in $\mathfrak{D}(L)$

$$
\left\|D_{j}^{2} f\right\| \leqq \gamma(\|L f\|+\|f\|) .
$$

This holds, in particular, on $\dot{\mathfrak{r}}_{2}$ and we see that if $L$ maps a Cauchy sequence $\left\{f_{n}\right\}$ into another Cauchy Sequence, then so does $D_{j}^{2}$. Thus relation (4.6) follows. Actually the novelty of Browder's estimate is the large class of regions $\mathscr{G}$ that he admits, over which the functions $f$ are defined. Since in our case $\mathscr{G}=\mathscr{E}_{n}$, the validity of $(4.7)$ on $\dot{\mathfrak{C}}_{2}$ could be derived from Aronszajn's original estimate [3], [38], if we remember the following: the boundedness of $\mathscr{G}$ was used only to establish the uniform continuity and ellipticity of the coefficients of $L$.

As a first consequence of relation (4.6) we see that the second resolvent equation applies to the operators $A$ and $L$. That is

$$
(i+L)^{-1}-(i+A)^{-1}=(i+A)^{-1} \cdot(L-A)(i+L)^{-1} \cdot
$$

As another consequence of relation (4.6), or rather of the coerciveness estimate (4.7) we shall derive that the second factor on the right of (4.8) is bounded. For, setting $f=(i+L)^{-1} g$ in (4.7) we obtain

$$
\left\|D_{j}^{2}(i+L)^{-1} g\right\| \leqq 2 \gamma\|g\|
$$


if we remember that since $L$ is self adjoint

$$
\|L f\|+\|f\| \leqq 2\|(i+L) f\| \text {. }
$$

Thus

$$
\left\|(L-A)(i+L)^{-1}\right\|<\infty .
$$

Insertion of this fact in relation (4.8) shows that

$$
M(p)\left[(i+L)^{-1}-(i+A)^{-1}\right] \equiv 0,
$$

since by assumption $p$ satisfies the conditions of Theorem 2.1, according to which

$$
M(p)(i+A)^{-1} \equiv 0 .
$$

Applying this fact again, relation (4.11) yields conclusion (4.4). Next we formulate:

THEOREM 4.2. Let the operator $L$ satisfy condition (4.2) and let the operator $A$ be defined by (4.3). Suppose that the function $p$ satisfies the conditions of Theorem 2.1. Then the operators $A$ and $L+M(p)$ are resolvent congruent, i.e.

$$
L+M(p) \equiv A \quad(i) .
$$

First we maintain that

$$
L \equiv A \quad(i) .
$$

To see this let $c_{k}$ be the characteristic function of the sphere of radius $k$. Then clearly

$$
\begin{aligned}
(i+L)^{-1} & -(i+A)^{-1} \\
= & (i+A)^{-1} M\left(c_{k}\right)(A-L)(i+L)^{-1} \\
& +(i+A)^{-1} M\left(1-c_{k}\right)(A-L)(i+L)^{-1} .
\end{aligned}
$$

Hence we see from Theorem 2.1 that

$$
(i+L)^{-1}-(i+A)^{-1} \equiv(i+A)^{-1} \cdot M\left(1-c_{k}\right)(A-L)(i+L)^{-1} .
$$

We claim that

$$
\lim _{k \rightarrow \infty}\left\|M\left(1-c_{k}\right)(A-L)(i+L)^{-1}\right\|=0 .
$$

For, we see from conditions (4.2) that there are functions $\left\{r_{j}^{(k)}\right\},\left\{s_{j}^{(k)}\right\}$, such that

$$
M\left(1-c_{k}\right)(A-L)=\sum_{j=1}^{n} M\left(r_{j}^{(k)}\right) D_{j}^{2}+\sum_{j=1}^{n} M\left(s_{j}^{(k)}\right) D_{j}
$$


and

$$
\lim _{k \rightarrow \infty}\left(\sup _{x} r_{j}^{(k)}(x)\right)=\lim _{k \rightarrow \infty}\left(\sup _{x} s_{j}^{(k)}(x)\right)=0 .
$$

Combining this relation with the coerciveness estimate (4.19), we see that

$$
\lim _{k \rightarrow \infty}\left\|\sum_{j=1}^{n} M\left(r_{j}^{(k)}\right) D_{j}^{2}(i+L)^{-1}\right\|=0 .
$$

Although we did not state it in (4.7) the coerciveness estimates also assert that - for operators more general than $L$-there is a constant $\gamma$ such that for every $f$ in $\mathfrak{D}(L)$,

$$
\left\|D_{j} f\right\|^{2} \leqq \gamma\left(\|L f\|^{2}+\|f\|^{2}\right) .
$$

This implies, in the same manner as (4.7) did imply (4.9), that

$$
\left\|D_{j}(i+L)^{-1}\right\|<\infty \text {. }
$$

Combining this relation with (4.19) we obtain

$$
\lim _{k \rightarrow \infty}\left\|\sum_{j=1}^{n} M\left(s_{j}^{(k)}\right) D_{j}(i+L)\right\|=0 .
$$

This in turn, combined with (4.18) establishes (4.15) in view of (4.16). Thus the operator on the left side of (4.14) is congruent to an operator of arbitrarily small norm and hence it is congruent to the 0 operator establishing relation (4.13).

Recall Theorem 4.1 which says that $M(p)$ is $L$-compact in the sense of Gokhberg-Krein. According to Lemma 1.1 it is a general operator theoretic fact that this implies the resolvent congruence property, i.e.

$$
L+M(p) \equiv L \quad(i) .
$$

Combining this relation with (4.13) we arrive at conclusion (4.12) and the proof of Theorem 4.2 is complete.

In conclusion, let us remark that the coerciveness inequality (4.19) is an elementary fact, since

$$
\left\|D_{j} f\right\|^{2}=\left(D_{j} f, D_{j} f\right) \leqq \gamma(f, L f) \leqq \gamma\|L f\|\|f\| \leqq \gamma\left(\|L f\|^{2}+\|f\|^{2}\right) .
$$

5. Perturbation of the Laplacian in an exterior region by a potential. Let $\mathscr{S}$ be a bounded hypersurface in $\mathscr{E}_{n}$, which divides it into two parts and denote the bounded interior by $\mathscr{G}_{b}$ and the unbounded exterior by $\mathscr{G}_{u}$. We define the unperturbed operator with the aid of the Laplacian acting in $\mathfrak{R}_{2}\left(\mathscr{G}_{u}\right)$ and with the aid of homogeneous boundary conditions. For the perturbation we take a potential 
acting in the same space $\mathscr{\Omega}_{2}\left(\mathscr{G}_{u}\right)$. We show that if the potential is locally bounded and satisfies the conditions of Theorem 2.1 then it defines a perturbation for which the Gokhberg-Krein property holds.

Specifically let $\mathscr{S}_{1}$ be some subsurface of $\mathscr{S}$, and let $\sigma$ be a continuous function on $\mathscr{S}_{1}$. Denote by $\mathfrak{S}(\sigma)$ the set of those twice continuously differentiable functions with bounded support in $\mathscr{G}_{u}$, which satisfy the boundary condition,

$$
\begin{aligned}
\frac{\partial f}{\partial n}(x)-\sigma(x) f(x) & =0 \quad x \in \mathscr{S}_{1} \\
f(x) & =0 x \in \mathscr{S}-\mathscr{S}_{1}
\end{aligned}
$$

$n$-being the outer normal on $\mathscr{S}$. Suppose that $\mathscr{S}$ is so smooth that the Gauss formula applies to it, i.e.

$$
\begin{aligned}
& (f,(-\Delta+M(p)) f) \\
& \quad=\int_{u} \sum\left(\frac{\partial f}{\partial x_{j}}\right)^{2} d x+\int_{u} p(x)|f(x)|^{2} d x+\int_{\mathscr{S}_{1}} \sigma|f(s)|^{2} d s, \\
& \quad \text { on } \mathfrak{D}(\sigma) .
\end{aligned}
$$

We shall also assume that $S$ is smooth enough to allow the following property $[47, \S 3][40]$, which we state for future reference: to any positive number $\varepsilon$ there is a number $\gamma(\varepsilon)$, such that

$$
\left.\left.\left|\int_{\mathscr{S}_{1}} \sigma\right| f(s)\right|^{2} d s\left|\leqq \varepsilon \int_{u_{j=1}}^{n}\right| \frac{\partial f}{\partial x_{j}}\right|^{2} d x+\gamma(\varepsilon) \int_{u}|f(x)|^{2} d x, \quad \text { on } \mathfrak{D}(\sigma) \text {. }
$$

Let $\Delta_{u}$ denote the Laplacian in $\mathfrak{D}(\sigma)$ in $\mathfrak{S}_{2}\left(\mathscr{G}_{u}\right)$. The essential self adjointness of this operator is far from being evident, as was the case with the corresponding free space operator. It follows from a result of Lax and Phillips [32], which says that for formally self adjoint elliptic boundary value problems-more general than ours-the weak solution is a strong solution. After these preparations we formulate:

THEOREM 5.1. Suppose that the real valued function $p$ is locally bounded and satisfies the conditions of Theorem 2.1. Then the operator $M(p)$ is $\Delta_{u}$-compact, i.e.

$$
M(p)\left(i+\Delta_{u}\right)^{-1} \equiv 0
$$

and

$$
\sigma_{e}\left(-\Delta_{u}+M(p)\right)=[0, \infty) .
$$

It is part of conclusion (5.4) that the operator on the left is 
defined on all of $\mathfrak{R}_{2}\left(\mathscr{G}_{u}\right)$. Actually all that we need, at present, is that it is densely defined; and this is the case since the set $\left(i+\Delta_{u}\right) \mathfrak{D}(\sigma)$ is dense. It is convenient to extend this operator to the larger space $\mathfrak{R}_{2}\left(\mathscr{E}_{n}\right)$ by setting

$$
p_{e}(x)= \begin{cases}0 & x \in \mathscr{G}_{b} \\ p(x) & x \in \mathscr{G}_{u}\end{cases}
$$

and

$$
\left(i+\Delta_{e}\right)^{-1}= \begin{cases}\left(i+\Delta_{b}\right)^{-1} & \text { on } \mathfrak{Q}_{2}\left(\mathscr{G}_{b}\right) \\ \left(i+\Delta_{u}\right)^{-1} & \text { on } \mathfrak{S}_{2}\left(\mathscr{G}_{u}\right) .\end{cases}
$$

Here $\Delta_{b}$ is the operator defined with the aid of $\Delta$ in $\mathscr{L}_{2}\left(\mathscr{C}_{b}\right)$ and a homogeneous boundary condition. The specific form of this boundary condition is not important since all of the operators $\left(i+\Delta_{b}\right)^{-1}$ are compact.

This extension is convenient inasmuch as the extended operator can be compared with the operator $M\left(p_{e}\right)\left(i+\Delta_{0}\right)^{-1}, \Delta_{0}$ being the Laplacian in free space. Specifically we see from Theorem 2.1 that conclusion (5.4) is equivalent to

$$
M\left(p_{e}\right)\left[\left(i+\Delta_{e}\right)^{-1}-\left(i+\Delta_{0}\right)^{-1}\right] \equiv 0 .
$$

We first maintain that if $w_{b}$ is a function which equals $I$ in and near $\mathscr{G}_{b}$ and vanishes near infinity, then

$$
M\left(w_{b}\right) M\left(p_{e}\right)\left[\left(i+\Delta_{e}\right)^{-1}-\left(i+\Delta_{0}\right)^{-1}\right] \equiv 0 .
$$

For, it was emphasized by Wolf, [47, § 12] that relations (5.2) and (5.3) imply that $\left(i+\Delta_{e}\right)^{-1}$ maps an arbitrary bounded set of functions in $\mathfrak{R}_{2}\left(\mathscr{E}_{n}\right)$, into a set of functions whose Dirichlet norm is bounded. From this and from the fact that the support of $w_{b}$ is bounded we conclude, using Rellichs' compactness criterion [17, vol. 1, Subsection VI. 2.2], that

$$
M\left(w_{b}\right)\left(i+\Delta_{e}\right)^{-1} \equiv 0 .
$$

Hence, in veiw of the local boundedness of $p_{e}$

$$
M\left(p_{e}\right) M\left(w_{b}\right)\left(i+\Delta_{e}\right)^{-1} \equiv 0 .
$$

Since evidently the function $w_{b} p_{e}$ satisfies the conditions of Theorem 2.1

$$
M\left(p_{e}\right) M\left(w_{b}\right)\left(i+\Delta_{0}\right)^{-1} \equiv 0,
$$

and relation (5.9) follows.

Next we set 


$$
w_{u}=1-w_{b}
$$

and maintain that

$$
M\left(p_{e}\right) M\left(w_{u}\right)\left[\left(i+\Delta_{e}\right)^{-1}-\left(i+\Delta_{0}\right)^{-1}\right] \equiv 0 .
$$

For, one expects that the operator $\left(i+A_{e}\right)^{-1}$ maps an arbitrary function in $\mathscr{S}_{2}\left(\mathscr{E}_{n}\right)$ into a function to which $\Delta$ is applicable locally, with the exception of the points of $\mathscr{S}$. In fact, according to Birmann [10, Lemma 1.3, p. 33], it is easy to show that for an arbitrary twice continuously differentiable bounded function which vanishes near $\mathscr{S}$, in particular for $w_{u}$,

$$
M\left(w_{u}\right)\left(i+\Delta_{e}\right)^{-1} \mathfrak{R}_{2}\left(\mathscr{E}_{n}\right) \subset \mathfrak{D}\left(i+\Delta_{0}\right) .
$$

Thus

$$
\begin{gathered}
M\left(p_{e}\right) M\left(w_{u}\right)\left[\left(i+\Delta_{e}\right)^{-1}-\left(i+\Delta_{0}\right)^{-1}\right]=M\left(p_{e}\right)\left(i+\Delta_{0}\right)^{-1} \\
\left(i+\Delta_{0}\right) M\left(w_{u}\right)\left[\left(i+\Delta_{e}\right)^{-1}-\left(i+\Delta_{0}\right)^{-1}\right],
\end{gathered}
$$

and according to Theorem 2.1 the first factor is compact. Therefore to complete the proof of relation (5.12) it suffices to show that

$$
\left\|\left(i+\Delta_{0}\right) M\left(w_{u}\right)\left[\left(i+\Delta_{e}\right)^{-1}-\left(i+\Delta_{0}\right)^{-1}\right]\right\|<\infty .
$$

Since the function $\left(i+\Delta_{e}\right)^{-1} f$ is essentially a Poisson integral the validity of this relation would follow from the integral representations for the first and second derivates of a Poisson integral [17, Subsection IV. 1.2]. Nevertheless, we prefer not to make use of these formulae and proceed differently. First we observe that since $w_{u}$ vanishes near $S$, there is a function $d$ which also vanishes near $\mathscr{S}$ and equals 1 on the support of $w_{u}$ : Hence

$$
M\left(w_{u}\right)=M(d) M\left(w_{u}\right)
$$

and

$$
M(d)\left(i+\Delta_{e}\right)^{-1} \mathscr{Q}_{2}\left(\mathscr{E}_{n}\right) \subset \mathfrak{D}\left(i+\Delta_{0}\right) .
$$

Insertion of relation (5.15) in (5.14) shows that the latter is equivalent to

$$
\left\|\left(i+\Delta_{0}\right) M\left(w_{u}\right) M(d)\left[\left(i+\Delta_{e}\right)^{-1}-\left(i+\Delta_{0}\right)^{-1}\right]\right\|<\infty .
$$

To see this, recall the well-known relation,

$$
\begin{aligned}
& \left(i+\Delta_{0}\right) M\left(w_{u}\right) \cdot M(d)=\sum M\left(\partial_{j}^{2} w_{u}\right) \cdot M(d) \\
& \quad+2 \sum M\left(\partial_{j} w_{u}\right) D_{j} \cdot M(d)+M\left(w_{u}\right)\left(i+\Delta_{0}\right) \cdot M(d),
\end{aligned}
$$

where we have set 


$$
\partial_{j} w_{u}(x)=\frac{\partial_{j} w_{u}}{\partial x_{j}}(x), \quad \partial_{j}^{2} w_{u}(x)=\frac{\partial^{2} w_{u}}{\partial x_{j}^{2}}(x),
$$

since $w_{u}$ is not in $\mathfrak{D}\left(D_{j}\right)$. Clearly the first term on the right of (5.16) is a bounded operator. In view of the coerciveness estimates [3] [38, Lemma 6] the second term is bounded with reference to $\left(i+\Delta_{e}\right)$, i.e.

$$
\left\|M\left(\partial_{j} w_{u}\right) D_{j} M(d)\left(i+\Delta_{e}\right)^{-1}\right\|<\infty \quad j=1,2, \cdots n .
$$

Finally we claim that

$$
M\left(w_{u}\right)\left(i+\Delta_{0}\right) M(d)\left[\left(i+\Delta_{e}\right)^{-1}-\left(i+\Delta_{0}\right)^{-1}\right]=0 .
$$

For, according to the second resolvent equation

$$
\left(i+\Delta_{e}\right)^{-1}-\left(i+\Delta_{0}\right)^{-1}=\left(i+\Delta_{e}\right)^{-1}\left(\Delta_{e}-\Delta_{0}\right)\left(i+\Delta_{0}\right)^{-1}
$$

holds on any set of functions which is mapped under $\left(i+\Delta_{0}\right)^{-1}$ into the intersection of the domains of $\Delta_{e}$ and $\Delta_{0}$. In particular this holds for any function $f$ in $\dot{\varepsilon}_{2}$, which can be written in the form $f=w_{u} g$. Since $\left(\Delta_{e}-\Delta_{0}\right)$ equals zere on the intersection of their domain, we have

$$
\left[\left(i+\Delta_{e}\right)^{-1}-\left(i+\Delta_{0}\right)^{-1}\right]\left(i+\Delta_{0}\right) M\left(w_{u}\right)=0 .
$$

Remembering the definition of the function $d$ and the local character of differentiation, this yields

$$
\left[\left(i+\Delta_{e}\right)^{-1}-\left(i+\Delta_{0}\right)^{-1}\right] M(d)\left(i+\Delta_{0}\right) M\left(w_{u}\right)=0 .
$$

Thus taking adjoints, at least formally, we have

$$
M\left(w_{u}\right)\left(i+\Delta_{0}\right) M(d)\left[\left(i+\Delta_{e}\right)^{-1}-\left(i+\Delta_{0}\right)^{-1}\right]=0
$$

and in view of the presence of the factor $M(d)$ this also follows rigorously. In other words, relation (5.18) has been established. Insertion of relations (5.16), (5.17), and (5.18) in $(5.14)_{d}$ shows the validity of this relation. This in turn establishes relation (5.11), which in view of (5.9) establishes (5.8) and conclusion (5.4) follows.

Having established this conclusion we derive conclusion (5.5). First observe that

$$
-\Delta_{u}+M(p) \equiv-\Delta_{u}(i) .
$$

For, we have seen that

$$
-\Delta_{e}+M\left(p_{e}\right) \equiv-\Delta_{e}(i)
$$

and according to definition (5.7) the operator $\Delta_{e}$ is the orthogonal sum of $\Delta_{u}$ and $\Delta_{b}$. Next we need the relation 


$$
\Delta_{e} \equiv \Delta_{0}(i)
$$

which is a special case of a result obtained independently by Glazman [23] and Wolf [47, $\$ 14$, Main Theorem]. It also follows from relation (5.18) and from the fact that Theorem 2.1 applies to the functions $\left\{\partial_{j} w_{u}\right\}$ and $\left\{\partial_{j}^{2} w_{u}\right\}$. For, a repetition of the arguments leading to (5.11) shows that this relation holds for the function $p_{e}(x) \equiv 1$, although Theorem 2.1 does not apply to this function. Since the essential spectrum of $\Delta_{b}$ is empty, relation (5.20) implies that

$$
\sigma_{e}\left(-\Delta_{u}\right)=[0, \infty]
$$

Finally combining this fact with relation (5.19) we arrive at conclusion (5.5), and the proof of Theorem 5.1 is complete.

In conclusion let us remark that this theorem is a slight generalization of a result obtained independently by Glazman [22] and Wolf [46], inasmuch as these authors required that the potential $p$ should be uniformly bounded and

$$
\lim _{|x| \rightarrow \infty} p(x)=0
$$

Added in Proof.

Agmon, S., Lectures on Elliptic Boundaryvalue Problems, Van Nostrand Co., 1966.

Schechter, M., On the invariance of the essential spectrum of an arbitrary operator, parts I and II. J. Math. Anal. Appl. 13 (1966), 205-215, and to appear.

Jorgens, K., UUber das wesentliche Spektrum elliptischer Differentialoperatoren vom Schrodinger-Typ, to appear.

REMARK to LEMMA 3.1. The assumption $P \geqq 0$ can be replaced by the assumption that the operator $A_{F}^{-1 / 2}|P| A_{F}^{-1 / 2}$ is compact. For, in this case inequality (3.2) holds on the complement of a finite dimensional subspace. Note that this assumption is equivalent to the compactness of $|P|^{1 / 2} A_{F}^{-1}|P|^{1 / 2}$.

\section{BIBLIOGRAPHY}

1. F. R. D. Agudo and F. Wolf, Proprietes spectrales des equations differentielles nonautoadjoints, Rend. Acad. Naz. Lincei 24, (1958), 643-645.

2.

$$
\frac{\partial^{2}}{\partial x^{2}}+\frac{\partial^{2}}{\partial y^{2}}+\frac{\partial^{2}}{\partial z^{2}}+a \frac{\partial}{\partial x}+b \frac{\partial}{\partial y}+c \frac{\partial}{\partial z}+d
$$

a coefficients complexes, Rend. Acad. Naz. Lincei. 25 (1958), 273-275.

3. N. Aronszajn, On coercive integro-differential quadratic forms, Conference on Partial Differential Equations, University of Kansas, 1954.

4. G. S. Avila and C. H. Wilcox, The propagation of time harmonic waves in homogenious anisotropic media, MRC Technical Summary Report (to appear). 
5. E. Balslev, The essential spectrum of elliptic differential operators in $L^{p}\left(R_{n}\right)$ (to appear, Trans. Amer. Math. Soc.)

6. - The essential spectrum of self adjoint elliptic differential operators in $L^{2}\left(R_{n}\right)$ (to appear, Math. Scand.)

7. - Perturbation of ordinary differential operators, Math. Scand. 11 (1962) 131-148.

8. - and T. W. Gamelin The essential spectrum of a class of ordinary differential operators, Pacific J. Math. (1964)

9. M. Sh. Birman, On the spectrum of singular boundary value problems, (Russian), Mat. Sbor. 97 (1961) 125-174. Math. Rev. 26 (1963), No. 463.

10. - Perturbations of continuous spectra of singular elliptic operators under the change of boundary and boundary conditions (Russian), Vestnik Leningradskovo Universiteta, Ser. Mat. Mech. Ast. (1962), 22-55. Math. Rev. 25 (1963) No. 2314.

11. and M. G. Krein, On the theory of wave operators, and scattering operators, Doklady Akad. Nauk SSSR 144 (1962), 475-478. Transl. Soviet Math. Dokl., 3, (1962), 740-747.

12. F. E. Browder, On the spectral theory of elliptic differential operators $I$, Math. Annalen 142 (1961), 22-130.

13. F. H. Brownell, A note on Cook's wave matrix theorem, Pacific J. Math. 12 (1962), 47-52.

14. - Perturbation of the n-dimensional Schrödinger differential operator, Bull. Amer. Math. Soc. (1954), No. 430.

15. - Spectrum of the static potential Schrödinger equation over $E_{n}$, Ann. of Math. 54 (1951), 554-594.

16. J. W. Calkin, Two sided ideals and congruences in the ring of bounded operators in Hilbert space, Ann. of Math. 42 (1941), 839-873.

17. Courant-Hilbert, Methods of mathematical physics, Vol. 2, Interscience Publishers, New York, 1962.

18. L. de Branges, Perturbations of self-adjoint transformations, Amer. J. Math. 84 (1962), 543-560.

19. N. Dunford and J. Schwartz, Linear operators Parts I and II, Chapters I-VIII and IX-XX respectively, Interscience Publishers, New York, 1957 and 1963.

20. K. O. Friedrichs, Spectral theory of operators in Hilbert space, N. Y. U. Lecture Notes, 1959-60.

21. Well posed differential equation problems in mathematical physics, N. Y. U. Lecture notes, 1963.

22. I. M. Glazman, On the application of the method of splitting to multidimensional singular boundary value problems, (Russian), Mat. Sbor. 35 (1954) 231-246. Math. Rev. 16 (1955), 827.

23. - On the spectra of ordinary, linear, singular boundary value problems, (Russian), Doklady Akad Nauk SSSR 87 (1952) 5-8. Math. Rev. 14 (1953), 1088.

24. I. C. Gokhberg and M. G. Krein, Basic propositions on deficiency numbers, root numbers, and indices of linear operators, (Russian), Upspehi Matem. Nauk 12 (1957), 43-118. Amer. Math. Soc. Transl. (2) 13 (1960) 185-264.

25. L. Hörmander, Estimates for translation invariant operators in $L^{p}$ spaces, Acta. Mat. 104 (1960) 95-140.

26. L Linear partial differential operators, Springer Verlag, 1963.

27. F. John, Plane waves and spherical means, Interscience Tracts in Pure and Appl. Math. No. 2, New York, 1955.

28. K. Jörgens, Wesentliche Selbstadjungiertheit singulärer elliptisher differentialoperatoren zweiter ordnung in $C_{0}^{\infty}(G)$, Math. Scand. 15 (1964) 5-17.

29. T. Kato, Fundamental properties of Hamiltonian operators of Schrödinger type, Trans. Amer. Math. Soc. 70 (1951) 196-211.

30. On the convergence of the perturbation method, Journal, Science Faculty, 
Univ. Tokyo, 1951.

31. - and T. Ikebe, Uniqueness of the self-adjoint extensions of singular elliptic differential operators, Archive Rat. Mech. Anal. 9, (1962), 77-92.

32. P. D. Lax and R. Phillips, Local boundary conditions for dissipative symmetric linear differential operators, Comm. Pure Appl. Math. 13 (1960) 427-455.

33. B. J. McLeod, On the continuous spectra of small potentials, (appear, Quart. J. Math., Oxford)

34. A. M. Molchanov, Discreteness criteria for self adjoint second order differential operators, (Russian), Trudy Mockovskovo Mat. Obshestvo. 2 (1953) 169-200.

35. P. A. Rejto, On the essential spectrum of the hydrogen energy operator, Math. Res. Ctr. Technical Summary Report 540, Madison, 1965.

36. F. Riesz and B. Sz. Nagy, Functional analysis, Translation, Frederick Ungar Co., New York, 1955.

37. M. Schechter, Invariance of the essential spectrum, Bull. Amer. Math. Soc. 71 (1965), 365-367.

38. - On estimating elliptic partial differential operators in the $L_{2}$ norm, Amer. J. Math. 79 (1957).

39. J. Schwartz, Some results on the spectra and spectral resolutions of a class of singular integral operators, Comm. Pure Appl. Math. 15 (1962) 75-90.

40. S. L. Sobolev, Application of functional analysis in mathematical physics. Translations of Math. Monographs, Vol. 7, A. M. S. 1963.

41. F. Stummel, Singuläre elliptische Differentialoperatoren in Hilbertschen Räumen, Math. Annalen 132 (1956), 150-176.

42. E. C. Titchmarsh, Eigenfunction expansions associated with second order differential equations, Clarendon Press, Oxford, 1946.

43. E. Wienholtz, Halbbeschränkte partielle differentialoperatoren zweiter ordnung vom elliptischen typus, Math. Annalen 135 (1958) 51.

44. F. Wolf, On the essential spectrum of partial differential boundary problems, Comm. Pure Appl. Math. 12 (1959), 211-228.

45. - On the invariance of the essential spectrum under a change of boundary conditions of partial differential operators, Proc. Konikl. Ned. Akad. Wet. (A) 62 (1959), 142-147.

46. - On the perturbation of an elliptic operator which leaves the essential spectrum invariant, Bull. Acad. Belg. 46 (1960) 441-447.

47. - On singular partial differential boundary problems, Ann. di Mat. Pura ed Applicada 69 (1960) 167-180.

48. B. Yood, Properties of linear transformations preserved under addition of a completly continuous transformation, Duke Math. J. 18 (1951) 599-612.

49. M. G. Zhiclin, On the spectrum of the many particle Schrödinger operator, (Russian), Trudy Mockovskovo Mat. Obshestvo. 9 (1960), 81-120. Math. Rev. 23A, No. 4023.

Received March 22, 1965, and in revised form September 29, 1965. This paper is an extension of the Technical Summary Report of [35], which was prepared under the auspices of the Mathematics Research Center, U. S. Army, the University of Wisconsin, Madison.

UNIVERSITY OF MINNESOTA 


\section{PACIFIC JOURNAL OF MATHEMATICS}

\section{EDITORS}

\author{
H. SAMELSON \\ Stanford University \\ Stanford, California \\ J. P. JANS \\ University of Washington \\ Seattle, Washington 98105
}

\author{
J. DUGUNDJI \\ University of Southern California \\ Los Angeles, California 90007 \\ RICHARD ARENS \\ University of California \\ Los Angeles, California 90024
}

\section{ASSOCIATE EDITORS}
E. F. BECKENBACH
B. H. NeumanN
F. WOLF
K. YOSIDA

\section{SUPPORTING INSTITUTIONS}

\author{
UNIVERSITY OF BRITISH COLUMBIA \\ CALIFORNIA INSTITUTE OF TECHNOLOGY \\ UNIVERSITY OF CALIFORNIA \\ MONTANA STATE UNIVERSITY \\ UNIVERSITY OF NEVADA \\ NEW MEXICO STATE UNIVERSITY \\ OREGON STATE UNIVERSITY \\ UNIVERSITY OF OREGON \\ OSAKA UNIVERSITY \\ UNIVERSITY OF SOUTHERN CALIFORNIA
}

STANFORD UNIVERSITY

UNIVERSITY OF TOKYO

UNIVERSITY OF UTAH

WASHINGTON STATE UNIVERSITY

UNIVERSITY OF WASHINGTON

AMERICAN MATHEMATICAL SOCIETY CHEVRON RESEARCH CORPORATION TRW SYSTEMS

NAVAL ORDNANCE TEST STATION 


\section{Pacific Journal of Mathematics}

\section{Vol. 19, No. 1 \\ May, 1966}

A. R. Brodsky, The existence of wave operators for nonlinear equations... 1

Gulbank D. Chakerian, Sets of constant width................... 13

Robert Ray Colby, On indecomposable modules over rings with minimum condition....................................... 23

James Robert Dorroh, Contraction semi-groups in a function space ....... 35

Victor A. Dulock and Harold V. McIntosh, On the degeneracy of the Kepler

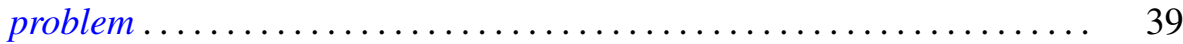

James Arthur Dyer, The inversion of a class of linear operators ......... 57

N. S. Gopalakrishnan and Ramaiyengar Sridharan, Homological dimension of Ore-extensions ................................. 67

Daniel E. Gorenstein, On a theorem of Philip Hall ................. 77

Stanley P. Gudder, Uniqueness and existence properties of bounded observables..................................... 81

Ronald Joseph Miech, An asymptotic property of the Euler function ....... 95

Peter Alexander Rejto, On the essential spectrum of the hydrogen energy and related operators ............................... 109

Duane Sather, Maximum and monotonicity properties of initial boundary

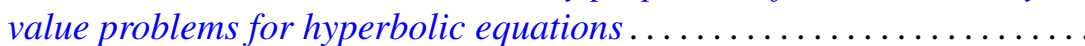

Peggy Strait, Sample function regularity for Gaussian processes with the parameter in a Hilbert space ........................... 159

Donald Reginald Traylor, Metrizability in normal Moore spaces ........... 175

Uppuluri V. Ramamohana Rao, On a stronger version of Wallis' formula ...............................

Adil Mohamed Yaqub, Some classes of ring-logics....... 